\title{
Lessons about food anaphylaxis
}

\author{
Author: Thomas Medveczky ${ }^{A}$
}

Exercise-induced anaphylaxis (EIA) and its subtype fooddependent (FD)-EIA are uncommon and easily missed forms of physical allergy. The latter is triggered by exercise following the ingestion of specific food products. Treatment is identical to that for IgE-mediated allergic reactions. The disease is potentially fatal. In this case, 30-year-old woman was seen in the allergy clinic at the request of her general practitioner. She reported an episode when she had gone to a local park to exercise after dinner. Shortly thereafter, she collapsed with rash, lip swelling, and breathing difficulties. Upon admission to hospital, she was found to be hypotensive and required fluid resuscitation, systemic corticosteroids and adrenaline. She made a full and uneventful recovery. On the basis of the clinical story and specific allergy markers, her presentation was attributed to FD-EIA.

KEYWORDS: Exercise-induced anaphylaxis, histamine, collapse, physical allergy, urticaria

\section{Case presentation}

A 30-year-old woman, who had previously been fit and well and with no history of diseases of the atopic diathesis (eczema, allergic rhinitis, food allergy or asthma) was referred to the allergy clinic after an episode of collapse associated with breathing difficulties shortly after dinner. She reported that on the day of the collapse, she invited friends for a meal. The dinner consisted of tapas, including dairy, pasta, prawns, and red wine. After the meal, she decided to go for a run in a nearby park. She left the house within 1.5 hours of eating and started to run gently. Within minutes, she felt unwell. She gave a description of developing a generalised pruritic rash followed shortly by lip swelling, which rapidly progressed to audible wheeze and breathing difficulties. A friend accompanying her in the park reported that she became pale and complained of colicky abdominal pain. Thereafter, she collapsed without losing consciousness. An ambulance was called, and upon arrival of the paramedics, she was found to be significantly cold, sweaty, and with low blood pressure, thus requiring rapid intravenous fluid challenge. She was transferred to a local emergency department, where further resuscitation

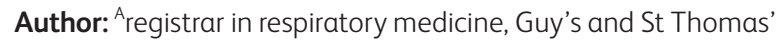
NHS Foundation Trust, London, UK with adrenaline, antihistamines and systemic corticosteroids was commenced. Her initial emergency presentation fulfilled the criteria for anaphylaxis (hypotension and respiratory compromise).

Her past medical history was unremarkable: specifically, she denied any symptoms of the atopic diathesis at any time in her life, and was taking no medications at the time of the incident. Her diet had previously been unrestricted.

She was stabilised and, on further questioning, physicians entertained the possibility that the reaction could have been linked with the previous consumption of her dinner. She made an uneventful recovery and was discharged 2 days later pending specialist allergist review.

\section{Investigations in clinic}

Scrutiny of the ingredient list from her dinner revealed three main food allergens: cow's milk, wheat and shellfish. Skin prick testing carried out in the allergy clinic offered possible diagnoses of an allergic reaction to $\omega$-5-gliadin (wheat) and/ or to prawns. Skin tests for both were positive according to international guidelines (ie they produced a wheal that was 3 $\mathrm{mm}$ bigger than that produced by the negative control (Table 1 ).

Because of the equivocal negative control, these tests were backed up by in vitro testing (ImmunoCAP ${ }^{\circledR}$ ). The ImmunoCAP ${ }^{\circledR}$ test revealed the presence of $\operatorname{IgE}$ antibodies specific for the $\omega$-5-gliaidin allergen at $0.03 \mathrm{kU} / \mathrm{ml}$ and for shellfish at $2.00 \mathrm{kU} / \mathrm{ml}$. This confirmed the elevated shellfishspecific sensitisation seen in the skin test.

She was advised to avoid heavy exercise soon after food and to avoid shellfish. Given the severity of her initial presentation, she was also trained in the use of an adrenaline injector. Her test results and the specific clinical picture were suggestive of food-dependent exercise-induced anaphylaxis

\section{Table 1. Results of skin prick testing.}

\section{Potential allergen} Size of wheal

Shellfish (mussels, clams, and prawns) $4 \mathrm{~mm}$

Wheat flour $4 \mathrm{~mm}$

Milk $2 \mathrm{~mm}$

\section{Eggs} $2 \mathrm{~mm}$

\section{Positive control} $5 \mathrm{~mm}$

Negative control 
(FD-EIA). This disease is easy to miss if not suspected (and there is considerable lack of awareness within the medical community), and is potentially fatal.

Fortunately, she recovered uneventfully with no further episodes.

\section{Discussion}

EIA is a unique form of a physical allergy characterised by rapid onset of generalised urticaria and occasionally complicated by respiratory compromise and/or cardiovascular collapse during or shortly after exercise. The first case report was published in 1979 by Maultiz et al ${ }^{1}$ and a second by Sheffer et $a l^{2}$ in 1983. EIA has since been described in association with the use of medications (such as aspirin and non-steroidal anti-inflammatory drugs (NSAIDs), which may also cause spontaneous urticaria and angioedema), and with premenstrual hormonal changes.

A variant of EIA that is perhaps more common in clinical allergy practice, although it has received scant attention in the literature, is anaphylaxis triggered by exercise soon after ingestion of food allergens. A clinical scenario emerged whereby patients would develop EIA following the intake of specific foodstuffs. This disease form is now termed FD-EIA. Episodes usually occur during exercise taken within hours of food ingestion and involve symptoms ranging from urticaria to systemic anaphylaxis. The first case report, which described an association with shellfish, was followed by a flurry of reports, in particular from Japan, of FD-EIA associated with wheat. Since then, further foods have been implicated in causing FD-EIA, including nuts (hazelnuts, almonds, peanuts and pistachio), vegetables (celery, onions, tomatoes, maize, matsutake mushrooms, lentils and chickpeas), fruits (pomegranate, grapefruit, apples, oranges, grapes and peaches), and some other types of food (snails, cuttlefish, tofu, egg, cow's milk and poppy seeds). ${ }^{3}$ Despite this, FD-EIA remains a rare and possibly underreported disease, with fewer than 1,000 cases in the literature: the largest observational study included 279 cases. The true prevalence may be higher.

Clinical records from a tertiary referral adult allergy clinic and training centre in London (Guy's and St Thomas' Hospital) suggest that 3-5 cases of EIA a month fit the definition of FDEIA. The few epidemiological studies that are available to date show a female predominance with a mean presentation age of 37 years. ${ }^{4}$ To date, one death has been described. ${ }^{5}$

In a large follow-up study of those with FD-EIA, 75\% of patients reported a family history of atopy, whereas 50\% reported a personal history of diseases of the atopic diasthesis. It should be noted, however, that some patients report neither a personal nor a family history of atopy, as in our case. In patients with FD-EIA, the time interval between ingesting food and the exercise and anaphylaxis reaction is 1-3 hours. ${ }^{6}$ During the event, up to $60 \%$ of patients described angioedema with breathing difficulties, whereas $30 \%$ had symptoms of cardiovascular collapse and abdominal cramps. ${ }^{7}$ Interestingly, a patient survey and long-term follow-up study by Shadick et al ${ }^{4}$ found that the frequency of attacks tends to stabilise or decrease with time. This may be a result of individual modification of exercise and avoidance of known environmental and ingestible precipitants.
The most important differential diagnoses in emergency settings include angioedema, acute severe asthma and cholinergic urticaria (CU). Angioedema would not be associated with cardiovascular collapse or urticaria. Acute severe asthma would of course be associated with wheeze and tachycardia, but urticaria and hypotension or cardiovascular collapse is not commonly associated with this presentation. An important differential diagnosis is CU, which is a form of urticaria that is typically associated with enhanced cholinergic activity in the skin, for example when sweating in hot environments or after exercise or other severe exertion.

The diagnosis of FD-EIA relies very much on clinical suspicion aroused by the appropriate clinical history and the presentation. The food trigger can often be identified or at least suspected from the history alone. The available literature reports that the most common food products involved are wheat and shellfish, but there are occasional reports implicating other products.

Apart from the history, clinical suspicion and appropriate testing for IgE sensitisation, some authors have proposed a 'gold standard' protocol to confirm the association between a food product and the risk of FD-EIA. This protocol takes the form of a double-blinded, placebo-controlled food exercise challenge. ${ }^{8}$ The patient is asked to ingest the suspected food allergen and then exercise on a treadmill according to a Bruce protocol. The value of this test is, however, limited with only a $70 \%$ positive predictive value, ${ }^{8}$ so a negative challenge does not rule out the diagnosis. In addition, the challenge test is labour-intensive and, like other diagnostic challenge scenarios in clinical allergy practice, potentially exposes the patient to risk; consequently, it is little used.

Skin prick tests using fresh foods or approved extracts should be performed on all patients. If they are negative when the diagnosis is strongly suspected, or if they are difficult to interpret, further in vitro tests for food-specific IgE antibodies may be helpful. In general, however, these serological tests remain less sensitive than skin prick tests, especially for fresh fruits, the allergens of which are particularly vulnerable to degradation during extraction. In cases where wheat is suspected, testing for IgE against $\omega$-5-gliadin has been helpful. These tests are usually performed using commercial, highintensity ELISA systems such as ImmunoCAP ${ }^{\circledR}$. As mentioned earlier, nut and grain allergens tend to be more robust than fruit and vegetable allergens for in vitro testing, but with this caveat, the correlation between skin testing and serology is generally good. Negative results with either test do not, however, exclude the condition. ${ }^{9}$

One proposed scenario for the pathophysiology of FD-EIA is 'mechanical' and centred around theories of: (a) exerciseinduced elevation of histamine release from mast cells and/ or basophils; (b) increased absorption of at least partially intact food allergens into the circulation in association with exercise; and (c) elevated blood flow in general, including increased splanchnic blood flow. Elevated circulating histamine is detectable after exercise even in 'healthy' subjects. It is suspected that this increase in mast cell releasability, combined with the better absorption of relatively intact allergen from the gut, which results from exercise soon after eating is sufficient to trigger systemic mast cell degranulation in some patients. ${ }^{10,11}$ 
Another hypothesis for the pathophysiology of FD-EIA proposes the formation of 'neoantigens' during exercise. Cross-linking of tissue transglutaminase (tTG) and the $\omega$-5-gliadin allergen found in wheat during exercise has been studied. ${ }^{12-14}$ Under 'normal' physiological conditions, tTG exists in an inactive biochemical state and functions as a signal-transducing G-protein, but under conditions of cellular damage or oxidative stress, which might be caused by exercise, this hypothesis proposes that elevated intracellular calcium might activate a cross-linking activity of tTG. At the same time, physical exercise elicits a subclinical inflammatory response manifested by increased levels of circulating proinflammatory cytokines, such as tumour necrosis factor $\alpha$ and other inflammation-responsive cytokines. This, in combination with an increased propensity of tTG to cross-link to $\omega$-5-gliadin, might result in the formation of a neoantigen that has much higher potency. ${ }^{15,16}$

In acute settings, the emergency management of FD-EIA is the same as that for any IgE-mediated systemic anaphylactic reaction: ${ }^{17}$ early administration of intramuscular adrenaline followed by fluid support, anti-histamines and bronchodilators if necessary. Systemic corticosteroids will mitigate against any delayed bronchospasm and angioedema. The cornerstones of successful longer-term management of FD-EIA are first to recognise the problem and second to educate the patient. Avoidance of culprit foods, preferably with the help of specialist advice from an allergy dietician, teaching the patient to recognise symptoms, to terminate exercise when necessary and to self-manage systemic episodes with adrenaline if necessary are crucial. In addition, previous studies frequently cite exercising during temperature extremes and humid conditions as particularly likely to provoke EIA. This should therefore be discouraged.

Once a link between the ingestion of a certain foodstuff and an exercise is established, the advice from most food experts would be to avoid any exercise up to 4 hours before and after ingestion of the foodstuff. Given the potential for systemic anaphylaxis, individuals who have been diagnosed with FD-EIA are advised to carry adrenaline autoinjector pens in addition to antihistamines.

Further research is required to identify possible targeted treatment options for FD-EIA patients. Currently, a UK-based study called TRACE is recruiting patients with peanut allergy to an attempt to identify the influence of external factors, including exercise, on their disease. Data analysis is still awaited and subject to ongoing trials. ${ }^{18}$

\section{Acknowledgements}

The author would like to thank the Allergists at Guy's Hospital (Drs Corrigan, Till and Haque) for their helpful comments.

\section{References}

1 Maulitz RM, Pratt DS, Schocket AL. Exercise-induced anaphylactic reaction to shellfish. J Allergy Clin Immunol 1979;63:433-4.
2 Sheffer AL, Soter NA, McFadden ER Jr, Austen KF. Exercise-induced anaphylaxis: a distinct form of physical allergy. J Allergy Clin Immunol 1983;71:311-6.

3 Perkins DN, Keith PK. Food- and exercise-induced anaphylaxis: importance of history in diagnosis. Ann Allergy Asthma Immunol 2002;89:15-23.

4 Shadick NA, Liang MH, Partridge AJ et al. The natural history of exercise-induced anaphylaxis: survey results from a 10-year followup study. J Allergy Clin Immunol 1999;104:123-7.

5 Flannagan LM, Wolf BC. Sudden death associated with food and exercise. J Forensic Sci 2004;49:543-5.

6 Sheffer AL, Tong AKF, Murphy GF et al. Exercise-induced anaphylaxis: a serious form of physical allergy associated with mast cell degranulation. J Allergy Clin Immunol 1985;75:479-84.

7 Sheffer AL, Austen KF. Exercise-induced anaphylaxis. J Allergy Clin Immunol 1980;66:106-11.

8 Aihara Y, Takahashi Y, Kotoyori T et al. Frequency of fooddependent, exercise-induced anaphylaxis in Japanese junior-highschool students. J Allergy Clin Immunol 2001;108:1035-9.

9 Romano A, DiFonso M, Guiffreda F et al. Food-dependent exercise-induced anaphylaxis: clinical and laboratory findings in 54 subjects. Int Arch Allergy Immunol 2001;125:264-72.

10 Sheffer AL, Tong AK, Murphy GF, Lewis RA, McFadden Jr ER, Austen KF. Exercise-induced anaphylaxis: a serious form of physical allergy associated with mast cell degranulation. J Allergy Clin Immunol 1985;75:479-84.

11 Horan RF, DuBuske LM, Sheffer AL. Exercise-induced anaphylaxis? Immunol Allergy Clin North Am 2001;21:769-82.

12 Palusuo K, Varjonen E, Nurkkala J et al. Transglutaminasemediated cross-linking of a peptic fraction of omega-5 gliadin enhances IgE reactivity in wheat-dependent, exercise-induced anaphylaxis. J Allergy Clin Immunol 2003;111:1386-92.

13 Ostrowski K, Rohde T, Asp S, Schjerling P, Pedersen BK. Pro- and antiinflammatory cytokine balance in strenuous exercise in humans. J Physiol 1999;515:287-91.

14 Pedersen BK, Hoffman-Goetz L. Exercise and the immune system: regulation, integration, and adaptation. Physiol Rev 2000;80: 1055-81.

15 Joint Task Force on Practice Parameters; American Academy of Allergy, Asthma and Immunology; American College of Allergy, Asthma and Immunology; Joint Council of Allergy, Asthma and Immunology. The diagnosis and management of anaphylaxis: an updated practice parameter. J Allergy Clin Immunol 2005;115(3 Suppl 2):S483-523.

16 Palosuo K, Alenius H, Varjonene E et al. A novel wheat gliadin as a cause of exercise-induced anaphylaxis. J Allergy Clin Immunol 1999;103:912-7.

17 Schwartz HJ. Elevated serum tryptase in exercise-induced anaphylaxis. J Allergy Clin Immunol 1995;95:917-9.

18 Royal Brompton Hospital London; Addenbrooks Hospital, University of Cambridge. TRACE peanut study. Available online at www.tracestudy.com/about [Accessed 5 August 2014].

Address for correspondence: Dr T Medveczky, St Thomas' Hospital, Westminster Bridge Road, London SE1 7EH, UK. Email: thomas-med@web.de 